\title{
Problems and Countermeasures of Healthy Personality Cultivation of Infants and Young Children in China
}

\author{
Yan Shunqin ${ }^{1}$ \\ ${ }^{1}$ School of education, China West Normal University, nanchong, China. \\ Correspondence: Yan Shunqin, School of education, China West Normal University, nanchong, China, ON., No 1 Shi \\ Da Road, Nanchong City, Sichuan Province, China.
}

Received: June 15, 2016

Accepted: June 29, 2016

Available online: July 13, 2016

doi:10.11114/ijsss.v4i8.1734

URL: http://dx.doi.org/10.11114/ijsss.v4i8.1734

\begin{abstract}
Infancy and early childhood (birth to 6 years old) is a critical period of personality formation. The perfection of personality during this period exerts inestimable profound influence on life-long personality development of the individual. The paper reviews current development and characteristics of personality of infants and young children in China, analyzes factors influencing personality formation and development, and problems in healthy personality development during this period, and in the end proposes some ways and methods to develop healthy personality during infancy and early childhood.
\end{abstract}

Keywords: infants and young children, healthy personality' personality development, ways, methods

Personality, a term with rich connotation, refers to a comprehensive system of unique and stable psychological characteristics of the individual on the biological basis, which is formed by and subject to social and living conditions. Healthy personality refers to a positive, harmonious and unified state of various aspects of an individual and between the individual and the social environment (Huang Junguan,2006).Freud emphasizes that the period of infancy and early childhood is vital for the formation and development of the individual's personality, maintaining that the structure of personality is basically formed in late infancy, which is the basis for future development of personality. Thus, the development of healthy personality in this period is of great significance to the personality health for the whole life of an individual.

\section{Introduction}

\subsection{Personality Development and Characteristics of Infants and Young Children in Current China}

Since the linguistic competence and comprehension of infants and young children is limited, their personality assessment is mainly conducted by their parents or kindergarten teachers based on their consistent and stable behaviors at home or in the kindergarten. According to parents' assessment of children's personality (questionnaires including five dimensions: extroversion, agreeableness, conscientiousness, emotional stability and exploratory ability), Zhang Tinghui finds children's personality develop well in other dimensions except conscientiousness, showing a positive feature; gender differences exist in agreeableness; five-year-olds perform remarkably better than three-year-olds in agreeableness and exploratory ability (Zhang Tinghui' 2009). Zhao Nan conducted a survey on young children's personality in Hunan province by using the "Teachers' Rating Questionnaire for Chinese Children Personality Development(3-12 years)" complied by Zhang Jinrong. The findings indicate that personality of young children in Hunan is generally in a medium level of development, with intelligence scoring high and emotional stability and pro-social behavior scoring on the low side; the personality of four-year-olds develops and changes most obviously; girls score slightly higher than boys in intelligence, earnest self-control and pro-social behavior while slightly lower than boys in extroversion and emotional stability (Zhao Nan' 2013).

\section{Factors Influencing Personality Development and Formation of Infants and Young Children}

The formation and development of infants' and young children's personality is mainly affected by two major factors( Zheng Ming, Xin Hongwei,2003): congenital heredity and acquired environment. Genetic factors lay the foundation for the formation while acquired environment plays a far more important and far-reaching role in personality formation and development. For infants and young children, the impact of the environmental factors in the development 
of their personality is mainly of family and kindergarten.

\subsection{The Influence of Family on the Formation and Development of infants' and young children's Personality}

Family is an important environment for the formation of a child's personality. Yin Liyang classifies the influence of family environment into two categories: subjective and objective factors. The former includes the personality and the perfection of personality of family members (especially parents), parenting style, emotional connection, mutual support and dialogue quality between family members; the latter, objective factors, refers to the composition of family members, domestic economic status, parents' educational level and occupation, social status of the family, basic life conditions of the family (food quantity and quality and accommodation conditions etc.)(Kalff AC,Kroes M\&Vles JS,2001). Studies show that children's behavioral problems are not only significantly related with their parents' occupation and educational level, but also closely related with the instability of family and intimacy among family members(Kalff AC,Kroes M\&Vles JS,2001). According to the study on children's behavioral problems conducted by YiMingji, a Chinese scholar, family harmony ensures the child's sense of security while family conflict entails the child's anxiety, stress and low self-esteem, which are harmful for the sound development of the his or her personality (Yi Ming Ji, Wei Shuzhen \& Zhang Yonghong, 1999) . The research of Xu Wei suggests that parental warmth and understanding, severe punishment or excessive interference substantially affects the child's temperament (Xu Wei, Zhu Yunxia \& Chen Ling, 2002) .Zheng Ming's investigation finds that the closeness between the child and parents as well as the emotional expression in the family have a negative correlation with the child's problematic behaviors (Zheng Ming \& Xin Hongwei, 2003).

\subsection{The Influence of Kindergarten on the Formation and Development of Personality}

Kindergarten is another important place for the formation and development of children' s personality. The teachers' personality perfection and the environment of the kindergarten play a leading role. The kindergarten teachers often have a greater authority than parents in the eyes of children. Children live, learn and play under the design and guidance of the kindergarten teachers. Therefore, the teachers are their guides, exerting great impact on the children's cognition, emotion, volition and behavior( Bao Xiulan, Zheng Yi\&Sun Shuying,2011). The kindergarten environment is the sum of all material and spiritual conditions required for children's physical and mental development, which has a stimulating effect on the cognitive development of young children. As a stimulus to children's development, the kindergarten environment can help kids cultivate certain behavioral habits, arouse their interest in learning and desire to explore. In addition, the kindergarten environment can also promote children's social ability.

\section{Problems in Healthy Personality Development of Infants and Young Children in China}

Long influenced by the deep-rooted thought that "Do not let children lose at the starting line", Chinese school education and family education have attached much more importance to knowledge teaching, learning achievement and enrollment rate than ability cultivation, moral quality and psychological health education. Therefore, the personality cultivation of infants and young children has been almost ignored, resulting in general problems among young children: poor independence, fragile psychology, fear of hardship and difficulty, being self-willed and inconsiderate, lack of creativity, little awareness and ability of social cooperation, and poor self-control ability, etc. Many children have a variety of psychological and behavioral biases, such as eccentric and aggressive behavior, timidness, hyperactivity, emotional disorder, etc (Mu Fengxia, Yu Yang, Dai Yaohua, etc., 2012; Liu Li, 2007).

In recent years, with increased educational levels of educators (including parents and kindergarten teachers), families and kindergartens have begun to realize the importance of healthy personality cultivation among infants and young children. Unfortunately, the situation is not so optimistic. The author thinks that there mainly exist the following problems:

First, the health level of educators' personality is to be improved.

In the general, the health level of parents' and even kindergarten teachers' personality is still low. For instance, they cannot keep themselves in perspective or understand the world in an objective way; they do not feel secure enough; they are unable to manage their own emotions; some are evennot capable of the job they take. Some educators have their own personality defects, obstacles even. These lead them to be impatient when instructing or interacting with kids, so they often discourage and criticize kids, damage their self-esteem and some even speak or behave rude towards kids, which will intangibly harm the kids' healthy growth, and the cultivation of their healthy personality. Therefore, it is extremely urgent to improve educators' own personality health level.

Second, educators' awareness of healthy personality development of young children needs to be improved.

Though aware of the importance of healthy personality of infants and young children, educators still have a superficial understanding of its development. First of all, they do not know the specific contents of personality cultivation, so they just do not know where to start in daily life or in education. Secondly, they are anxious for success. Therefore, educators 
need to understand the contents of healthy personality development in infants and young children. Healthy personality means that various psychological aspects of the young kids are in a positive and agreed coordination with social environment, including the following contents: sense of security; self-belief and self-acceptance, trust in others; having some hobbies and lively and cheerful personality; emotional stability and proper expression of emotion; curiosity about the world; respect for others and ability to get along with people; being brave, generous and helpful, ability to cooperate and share with others; being strong-willed, self-disciplined; having one's own ideas and opinions; having goals in doing things (Huang Yongfang \& Yu Wei, 2005) and so on. It is because of the above characteristics in healthy personality cultivation of infants and young children that healthy personality training and shaping is a systematic project which cannot be completed overnight. Thus, educators are supposed to be loving, patient, persevering and unyielding, and they need to continue to explore new scientific strategies for children's healthy personality development.

\section{Ways and Methods to Develop Healthy Personality of Infants and Young Children}

The cultivation of healthy personality in infants and young children mainly includes two parts: family education and kindergarten education. Then, through what approaches and ways can the family and kindergarten cultivate healthy personality of infants and young children?

First, to establish a secure parent-child attachment. This is conducive for children to have faith in the world, others and themselves, and it lays a solid foundation for the individual to establish interpersonal and intimate relationship in the future. Secure parent-child attachment also has a direct impact on children's establishment of self concept, formation of moral judgment and healthy development of individuality.

Second, to create a harmonious family atmosphere and democratic parenting style. The harmonious atmosphere of family is helpful for children to form positive, initiative and optimistic character. Studies show that parental rearing pattern is closely related with the development of children's personality. Appropriate severity and love of parents (both expectations and praises) enables childern to put themselves in perspective and evaluate themselves, build self-esteem and confidence. Conversely, children will be lacking in a sense of security, unable to trust people, and even behave aggressive or violent ( Zhang Tinghui, 2009).

Third, to respect children, protect their self-esteem and enhance their self-confidence. Parents and teachers should treat children on an equal footing, respect their wishes, reasonable needs, interests and hobbies, and think more often in children's shoes. In daily life, parents and teachers should give children more opportunities to make their own choices and decisions. Don't protect or restrict them too much, but have full faith in their own ability.

Fourth, to set a role model for infants and young children to follow. The development of children's personality depends more on the influence of what they constantly see and hear than on education. Educator Herbert Spencer said: "Brutality begets brutality; love generates love"( Huang Junguan \& He Fangqing, 2008). Parents and teachers are models in the eyes of infants and young children, who exert profound influence on their behavior. Since young children are very good at observing and imitating, educators must take their own noble characters as an example for children, so as to influence their speech, behavior, habits, character, style and bearing, etc., which in turn influence children and then their personality development.

Fifth, to improve personality health of kindergarten teachers and parents. Teachers' personality health is an important guarantee of cultivating healthy personality for infants and young children. First of all, starting from the normal education, let future educators deepen their understanding of healthy personality and improve their own personality. At the same time, strengthen the in-service teachers' continuing education, promote their educational level, and meanwhile alleviate their psychological pressure and solve their psychological problems, so that each and every educator can make a positive contribution to developing students' healthy personality. In addition, actively carry out school-family cooperation. Schools spread regularly knowledge about health personality to parents and provide them with necessary psychological counseling. Improve parents' education and cultural quality, help them shape good personality traits so that they can guide and help children in life, and thus cultivate children's healthy personality.

\section{Conclusion}

Healthy personality development of infants and young children is of great significance to their mental health; personality perfection during infancy and early childhood greatly influences the future construction of personality for the individual. To this end, family, school and society should cooperate with each other in developing healthy personality of infants and young children which can ensure the promotion of healthy personality development.

\section{References}

Bao, X. L., Zheng, Y., ... Sun, S. Y. (2011). A Study on the Cultivation of Sound Personality of Infants and Young Children with the Comprehensive Intervention in the Early Period of 0-3 years . Journal of Chinese Children Health Care, 19(7), 606-609. 
Huang, J. G. (2006). On the Development of Healthy Personality of the Single Child, Education and Occupation, 18, 87-89.

Huang, J. G., \& He, F. Q. (2008). On the Cultivation of Healthy Personality of Young children. Journal of Yulin Normal University (philosophy and social sciences), 29(6), 116-120.

Huang, Y. F., \& Yu, W. (2005). On Strategy of Young Children's Healthy Personality Development.Education Forum, $8,37-38$.

Kalff, A. C., Kroes, M., ... Vles, J. S. (2001). Factors affecting the relation between parental education as well as occupation and problem behaviour in dutch 5 to 6-year-old children. Soc Psychiatry Epidemiol, 36(7), 324-331. http://dx.doi.org/10.1007/s001270170036

Kalff, A. C., Kroes, M., ... Vles, J. S. (2001). Neighbourhood level and individual level SES effects on child problem behavior amultilevel analysis. Epidemiol Community Health, 55(4), 246-250. http://dx.doi.org/10.1136/jech.55.4.246

Liu, L. (2007). Reflections on the Shaping of Children's Healthy Personality.Educational and Teaching Research, 7, 185-186.

Mu, F. X., Yu, Y, ... Dai, Y. H. (2012). Effect of Early Intervention on the Development of Social Emotion of Infants and Young Children . Research on the Health of Chinese Women and Children, 23(4), 413-415;

Xu, W., Zhu, Y. X., \& Chen, L. (2002). Family Environment and Children's Temperament. Chinese Journal of Mental Health, 16(6), 414.

Yi, M. J., Wei, S. Z., \& Zhang, Y. H. (1999). The Influence of Family Mental Environment on Children's Behavior, Chinese Journal of Pediatrics, 37(3), 146.

Zhang, T. H. (2009). Research on the Influence of Family Environment on the Development of Children's Personality. Yangtze Normal University, 25(3), 152-155.

Zhao, N. (2013). Status and Characteristics of Personality Development of Children Aged 3 to 6in Hunan Province, Chinese Special Education, 8, 90-96.

Zheng, M., \& Xin, H. W. (2003). Study on the Behavioral Problems of Children Aged 4 to 6 and Family Psychological Environment in Lanzhou, Journal of Health Psychology, 11(6), 451-453.

\section{(cc) $\mathrm{Br}$}

This work is licensed under a Creative Commons Attribution 3.0 License. 